\title{
Cast Nanostructured Films of Poly(methyl methacrylate-b-butyl acrylate)/Carbon Nanotubes: Influence of Poly(butyl acrylate) Content on Film Evaporation Rate, Morphology, and Electrical Resistance
}

\author{
F. Soriano-Corral, L. F. Ramos-de Valle, F. J. Enríquez-Medrano, P. A. De León-Martínez, \\ M. L. López-Quintanilla, and E. N. Cabrera-Álvarez
}

Polymer Processing Department, Centro de Investigación en Química Aplicada (CIQA), Boulevard Enrique Reyna, No. 140, Colonia San José de los Cerritos, 25294 Saltillo, COAH, Mexico

Correspondence should be addressed to F. Soriano-Corral, fsoriano@ciqa.mx

Received 28 February 2012; Accepted 24 March 2012

Academic Editor: Sevan P. Davtyan

Copyright (C) 2012 F. Soriano-Corral et al. This is an open access article distributed under the Creative Commons Attribution License, which permits unrestricted use, distribution, and reproduction in any medium, provided the original work is properly cited.

\begin{abstract}
Nanocomposites of poly(methyl methacrylate-b-butyl acrylate)/multiwalled carbon nanotubes were prepared from different copolymers synthesized by RITP technique using iodine functionalized poly(methyl methacrylate) as macrochain transfer agent to obtain block copolymers with butyl acrylate as comonomer in a sequential copolymerization. Poly(butyl acrylate) contents of 7, 20, and $30 \mathrm{wt} \%$ were attained in each copolymer. These copolymers were used to prepare nanostructured films by casting process, using chloroform as solvent, and carboxyl functionalized MWCNT at 0.4, 0.6, 0.8, 1.0, and 1.2 wt\%. During the film preparation, the absolute drying rate $(N)$ was calculated with respect to the poly(butyl acrylate) and MWCNT composition. For copolymers containing 7 and $20 \mathrm{wt} \%$ of poly(butyl acrylate) the $N$ values slightly decrease with the MWCNT concentration, while for the suspension prepared with the copolymer at $30 \mathrm{wt} \%$ of poly(butyl acrylate) the $N$ values decrease drastically down to $50 \%$ approximately. The MWCNT content at the percolation threshold point was found to be $0.8 \mathrm{wt} \%$, for all nanostructured films. The dispersion of MWCNT within the polymer matrix decreased with increasing the poly(butyl acrylate) composition, but it did not affect the electrical properties, which is assumed to be due to induction of the bridging effect and the MWCNT preference to locate into the poly(methyl methacrylate) phase.
\end{abstract}

\section{Introduction}

Synthesis of carbon nanotubes (CNTs) was firstly reported by Iijima [1] in the 1990s. CNT present a cylindrical structure and they can be single walled (SWCNT) or multi walled (MWCNT) with an average diameter oscillating between 1 and $50 \mathrm{~nm}$. The incorporation of SWCNT and/or MWCNT in polymeric and/or ceramic materials can result in an improvement of some specific properties with respect to the virgin materials. Properties like mechanical performance in polypropylene, polycarbonate [2], and polyethylene terephthalate (PET) [3]; crystallization rate in poly (L-lactide) [4]; surface electric conductivity in polydimethylsiloxane [5] and PET [6]; and heat transfer in ethylene-vinyl acetate copolymer (EVA) [7] can be increased when CNTs are efficiently incorporated. Concentrations of $0.5-2 \mathrm{wt} \%$ of CNT provoke electric conductivity in several polymers $[2,5,6]$. Chemical surface modification of CNT is frequently carried out through the addition of hydroxyl or carboxylic acid functionalities allowing a stronger physical interaction between CNT and the polar polymeric matrices. This functionalization will facilitate the CNT dispersion and reduce the amount required to produce a given improvement in properties. Khosla and Gray [6] reported that the percolation threshold—for resistivity-in polydimethylsiloxane/CNT compounds, occurred at $2 \mathrm{wt} \%$ when using nonmodified CNT, whereas it occurred at $1.5 \mathrm{wt} \%$ when using carboxyl-modified CNT. In a study of PET/CNT compounds, Cruz-Delgado et al. [5] reported 
TABLE 1: Reaction conditions for the synthesis of macrochain transfer agents and block copolymers by RITP.

\begin{tabular}{lcccc}
\hline Experiment & MMA $(\mathrm{g})$ & AIBN $(\mathrm{g})$ & $\mathrm{I}_{2}(\mathrm{~g})$ & \\
\hline 1 & 45 & 0.11 & 0.09 & $\mathrm{BuA}(\mathrm{g})$ \\
2 & 35 & 0.11 & 0.09 & 5 \\
3 & 25 & 0.11 & 0.09 & 15 \\
\hline
\end{tabular}

Reaction time for PMMA block $=12 \mathrm{~h}$, reaction time for PBuA block $=12 \mathrm{~h}, T=70^{\circ} \mathrm{C},[\mathrm{AIBN}] /\left[\mathrm{I}_{2}\right]=1.9$, and $[\mathrm{AIBN}] /[\mathrm{PMMA}]=0.3$.

TABLE 2: Results of the synthesis of macrochains transfer agents and block copolymers by RITP.

\begin{tabular}{lcccccccccc}
\hline & \multicolumn{3}{c}{ Step 1. Synthesis of PMMA } & \multicolumn{5}{c}{ Step 2. Copolimerization PMMA- $b$-PBuA } \\
Exp & ${ }^{\mathrm{a}} \mathrm{X}_{\text {PMMA }}$ & ${ }^{\mathrm{b}} \mathrm{Mn}_{\text {Th1 }}$ & ${ }^{\mathrm{c}} \mathrm{Mn}_{\text {PMMA }}(\mathrm{g} / \mathrm{mol})$ & ${ }^{\mathrm{d}} \mathrm{I}_{\text {PMMA }}$ & $\mathrm{X}_{\text {PMMA }}$ & $\mathrm{X}_{\text {PBuA }}$ & ${ }^{\mathrm{e}} \mathrm{Mn}_{\text {Th2 }}$ & ${ }^{\mathrm{f}} \mathrm{Mn}_{\text {Copol }}(\mathrm{g} / \mathrm{mol})$ & $\mathrm{I}_{\text {Copol }}$ & ${ }^{\mathrm{g} P M M A}:$ PBuA \\
\hline 1 & 0.85 & 54000 & 44500 & 1.4 & 0.99 & 0.70 & 56000 & 48000 & 1.6 & $93: 7$ \\
2 & 0.89 & 44000 & 29000 & 1.6 & 0.99 & 0.60 & 41000 & 38000 & 1.9 & $80: 20$ \\
3 & 0.90 & 32000 & 24000 & 1.8 & 0.99 & 0.44 & 38000 & 31000 & 1.9 & $70: 30$ \\
\hline
\end{tabular}

${ }^{\mathrm{a}} \mathrm{X}$ is the fractional conversion; ${ }^{\mathrm{b}} \mathrm{Mn}_{\mathrm{Th} 1}$ is the theoretical Mn for PMMA macrochain transfer agent; ${ }^{\mathrm{c}} \mathrm{Mn}$ PMMA is the experimental Mn for PMMA; ${ }^{\mathrm{d}}$ is the polydispersity index; ${ }^{\mathrm{e}} \mathrm{Mn}_{\mathrm{Th} 2}$ is the theoretical $\mathrm{Mn}$ for the copolymer; ${ }^{\mathrm{f}} \mathrm{Mn}_{\mathrm{Copol}}$ is the experimental $\mathrm{Mn}$ for the copolymer; ${ }^{\mathrm{g}} \mathrm{PMMA}$ : $\mathrm{PBuA}$ is the PMMA and $\mathrm{PBuA}$ composition calculated by ${ }^{1} \mathrm{H}$ NMR.

conductivity values of $1 \times 10^{-12} \mathrm{~S} / \mathrm{cm}$ when using $1 \mathrm{wt} \%$ of hydroxyl modified CNT, whereas it was cero when using nonmodified CNT. This was attributed to the much better dispersion when using modified CNT. In all cases, however, the addition of CNT into a polymer matrix will increase the modulus, which could be a problem in applications where flexibility and comfort are needed, such as in electrostatic dissipative (ESD) coatings on textile fibers. It is at this point where design of polymer matrices (nature and structure) becomes an important factor to consider, in order to obtain a polymer nanocomposite with desirable properties such as a flexibility and conductivity. The choice of the polymerization method, to obtain homopolymers or copolymers, becomes of great relevance.

Reversible deactivation radical polymerization (RDRP) techniques provide novel routes to synthesize well-defined low-polydispersity block copolymers and other types of complex architectures [8-10]. The most popular RDRP techniques are nitroxide-mediated polymerization (NMP) [11], atom transfer radical polymerization (ATRP) [12], and reversible addition-fragmentation chain transfer polymerization (RAFT) [13]. A newer and simpler technique, known as reverse iodine transfer polymerization (RITP) $[14,15]$, relies on the use of molecular iodine to control (and confer functionality) the polymerization. The low cost of molecular iodine and the simplicity of the technique (the chain transfer agents are synthesized in situ at the beginning of the polymerization) are enormous advantages over other techniques. Synthesis of different types of block copolymers using RITP have been reported [16-18] demonstrating the efficiency of this technique, regulated by a degenerative transfer mechanism. The controlled synthesis of poly(methyl methacrylate) (PMMA) by RITP in toluene has been reported by Lacroix-Desmazes and coworkers [19-21], attaining molecular weights between 5000 and $20000 \mathrm{~g} / \mathrm{mol}$.

Here we report the synthesis of three different materials of iodine-functionalized PMMA by RITP, targeting higher molecular weights than those previously reported [19]. Synthesis of block copolymers using the PMMA as macrochain transfer agents and butyl acrylate are also reported.
Thereafter, we studied the effect of poly(butyl acrylate) $(\mathrm{PBuA})$ content on the drying rate, morphology, and the electric properties of nanocomposite films constituted of the copolymer poly(methyl methacrylate- $b$-butyl acrylate) and carboxyl-modified carbon nanotubes using chloroform $\left(\mathrm{CHCl}_{3}\right)$ as solvent.

\section{Experimental}

2.1. Materials. Methyl methacrylate (MMA) and n-butyl acrylate $(\mathrm{BuA})$ were purified by vacuum distillation before use. 2,2'-azobis(isobutyronitrile) (AIBN) was recrystallized from ethanol. Toluene was distilled before use and molecular iodine $\left(\mathrm{I}_{2}\right)$ was used as received. All the aforementioned substances were from Aldrich, while the carboxyl-modified MWCNTs, with $0.5-3 \mathrm{wt} \%$ of $\mathrm{COOH}$, average diameter of $30-50 \mathrm{~nm}$, and length of $15-20 \mu \mathrm{m}$, were from AlphaNano Technology Co. LTD.

2.2. Synthesis of Copolymers. The procedure for the copolymers synthesis is exemplified following experiment number 1 from Table $1 ; 45 \mathrm{~g}(0.45 \mathrm{~mol})$ of MMA, $45 \mathrm{~g}$ $(0.489 \mathrm{~mol})$ of toluene, $0.090 \mathrm{~g}(0.354 \mathrm{mmol})$ of $\mathrm{I}_{2}$, and $0.110 \mathrm{~g}(0.670 \mathrm{mmol})$ of AIBN were introduced in a round flask. The mixture was fluxed with argon for 30 minutes, after which the flask was placed in an oil bath at $70^{\circ} \mathrm{C}$. The polymerization was conducted for 12 hours in the dark, with magnetic stirring and under argon atmosphere. Conversion (X) was determined via ${ }^{1} \mathrm{H}$ NMR analysis on a crude sample of iodine functionalized PMMA and molecular weight distribution was determined by size exclusion chromatography $(\mathrm{SEC})$. Results obtained were $X_{\mathrm{PMMA}}=$ $0.85, \mathrm{Mn}_{\text {PMMA }}=44500 \mathrm{~g} / \mathrm{mol}$, and $\mathrm{Mw} / \mathrm{Mn}_{\text {PMMA }}=1.4$ (see Table 2 for the complete results). In the flask containing the crude sample (PMMA), $5 \mathrm{~g}(0.039 \mathrm{~mol})$ of BuA, $5 \mathrm{~g}$ $(0.054 \mathrm{~mol})$ of toluene, and $0.042 \mathrm{~g}(0.257 \mathrm{~mol})$ of AIBN were added. In all cases, an $[\mathrm{AIBN}] /[\mathrm{PMMA}]=0.3$ was used. Again, the mixture was fluxed with argon for 30 minutes and the flask was placed in an oil bath at $70^{\circ} \mathrm{C}$. The 
polymerization was conducted for 12 hours in the dark, with magnetic stirring and under argon atmosphere, resulting in a poly(methyl methacrylate)- $b$-poly(butyl acrylateco-methyl methacrylate). Conversion (X) was determined via ${ }^{1} \mathrm{H}$ NMR analysis on a crude sample of copolymer and molecular weight distribution was determined by size exclusion chromatography (SEC). Results obtained were $\mathrm{X}_{\mathrm{PBuA}}=0.70, \mathrm{Mn}_{\text {Copol }}=48,000 \mathrm{~g} / \mathrm{mol}$, and $\mathrm{Mw} / \mathrm{Mn}_{\text {Copol }}=1.6$ (see Table 2 for the complete results). Experiments 2 and 3 were conducted in a similar way, adjusting the monomer mass ratio.

2.3. Characterization of Copolymers. Molecular weights of polymer samples were determined by size exclusion chromatography (SEC) using a Hewlett-Packard instrument (HPLC series 1100) equipped with a refractive index detector. A PLGel-mixed column was used. Calibration was carried out with polystyrene standards and THF (HPLC grade from Sigma-Aldrich) was used as eluent at a flow rate of $1 \mathrm{~mL} / \mathrm{min}$.

${ }^{1} \mathrm{H}$ nuclear magnetic resonance (NMR) spectra of polymer samples were obtained with a JEOL Eclipse-300 MHz spectrometer, using $\mathrm{CDCl}_{3}$.

Morphology of the copolymers was observed in a TITAN transmission electron microscope (TEM). Previously, the compression-molded samples were cut cryogenically at $-32^{\circ} \mathrm{C}$ and a cut rate of $2 \mathrm{~mm} / \mathrm{min}$, in slices of $50 \mathrm{~nm}$ thick using an LEICA ultra-microtome, after which the samples were stained using ruthenium tetraoxide $\left(\mathrm{RuO}_{4}\right)$ vapors to contrast the PBuA phase in the copolymers [22].

Thermal properties of copolymers were analyzed using a V4.3A TA Instruments differential scanning calorimeter (DSC) from -50 to $150^{\circ} \mathrm{C}$ at $5^{\circ} \mathrm{C} / \mathrm{min}$.

2.4. Nanostructured Films Preparation and Characterization. $0.5 \mathrm{~g}$ of the synthesized copolymers was dissolved in $20 \mathrm{~mL}$ of chloroform and carboxyl-modified MWCNTs at 0.4, 0.6, 0.8 , and $1 \mathrm{wt} \%$ were added to form colloidal suspensions. The mixing of colloidal suspensions was made by means of ultrasound at a frequency of $40 \mathrm{kHz}$ for 1 minute at room temperature $\left(25^{\circ} \mathrm{C} \pm 2\right)$. The colloidal suspensions were deposited on Pyrex glass coverslips, from Aldrich, in an analytical balance and the drying rate $(N)$ was evaluated according to (1):

$$
N=\frac{L s}{A}\left(-\frac{d w}{d t}\right),
$$

where $N$ is the drying rate; Ls is the dry mass; $A$ is the evaporation area; $d w / d t$ is the mass loss through evaporation as a function of time [23].

The storage modulus of the neat copolymers and nanostructured films was evaluated by means of DMA Q800 dynamical mechanical analyzer in strain mode at a frequency of $1 \mathrm{~Hz}$, from -50 to $150^{\circ} \mathrm{C}$ at $5^{\circ} \mathrm{C} / \mathrm{min}$.

The surface electrical resistance of the prepared films was measured using an ACL 390 Staticide surface resistance meter, and the percolation threshold concentration of MWCNT was established.

To analyze the dispersion of MWCNT in the copolymers matrices, a JEOL scanning electron microscope (SEM) was used.

\section{Results and Discussion}

3.1. Synthesis of Copolymers. In the RITP mechanism the $\mathrm{I}_{2}$ reacts with fragments of the initiator or with low molecular weight species to generate iodinated chain transfer agents. The consumption of monomer in this step is low and it is called the inhibition period. Once all molecular iodine is consumed, the polymerization period takes place. Three experiments were performed by RITP to produce PMMA with different molecular weights. Polymerizations were carried out in toluene ( $50 \mathrm{wt} \%$ ), using AIBN as initiator. A molar ratio $\mathrm{AIBN} / \mathrm{I}_{2}=1.9$ was used, which is common in an RITP experiment. Table 2 shows the obtained results for the synthesis section. During the PMMA syntheses high conversions were observed and polydispersity indexes around 1.6 were similar to results reported for RITP of MMA in solution [19]. Conversions close to 0.90 are advantageous to avoid a purification step and continue directly with the formation of the second block since small amount of residual monomer should not severely modify the properties of the second PBuA block. According to;

$$
\begin{aligned}
\mathrm{Mn}= & \frac{(\mathrm{g} \text { of monomer })(\text { conversion })}{2\left(\text { moles of } \mathrm{I}_{2}\right)} \\
& +M W_{\text {chain-ends }},
\end{aligned}
$$

the expected Mn of PMMA in experiment 1 was $54000 \mathrm{~g} / \mathrm{mol}$, while the experimental value was determined as $44500 \mathrm{~g} / \mathrm{mol}$ based on a polystyrene calibration curve. This difference between the experimental and theoretical $\mathrm{Mn}$ is because of the high targeted molecular weight. An adequately controlled RDRP technique is guaranteed when the targeted molecular weight is below $20000 \mathrm{~g} / \mathrm{mol}$. However, considering the high molecular weights required for this work, this insufficiently adequate control was disregarded. Theoretical Mn for experiments 2 and 3 (synthesis of PMMA) was 44000 and $32000 \mathrm{~g} / \mathrm{mol}$, respectively.

Sequential step block copolymerizations of $\mathrm{BuA}$ as monomer- and iodine-functionalized PMMA as macrochain transfer agent were carried out. Thereafter, the prepared PMMAs were used for the synthesis of block copolymers of poly(methyl methacrylate)- $b$-poly(butyl acrylate-co-methyl methacrylate). In experiments 1,2 , and 3 , the $\mathrm{Mn}$ was increased by growing the second block. In experiment 1 , for example, the Mn attained after the synthesis of the diblock copolymer was $48000 \mathrm{~g} / \mathrm{mol}$, whereas the Mn after the MMA homopolymerization was $44500 \mathrm{~g} / \mathrm{mol}$. Theoretical Mn for three different copolymers adjusted acceptably with the 
TABLE 3: Parameters used to calculate prediction of $T_{g}$ of soft segments by the Fox equation.

\begin{tabular}{lcccccc}
\hline Experiement & ${ }^{\mathrm{a}} \mathrm{F}_{1}$ & ${ }^{\mathrm{b}} 1-\mathrm{F}_{1}$ & $T_{g, 1=\text { PBuA }}\left({ }^{\circ} \mathrm{C}\right)$ & $T_{g, 2=\text { PMMA }}\left({ }^{\circ} \mathrm{C}\right)$ & $T_{g \text { Fox }}\left({ }^{\circ} \mathrm{C}\right)$ & $T_{g \text { Experimental }}\left({ }^{\circ} \mathrm{C}\right)$ \\
\hline 1 & 0.3437 & 0.6562 & -52 & 100 & 28 & 90 \\
2 & 0.7024 & 0.2975 & -52 & 100 & -21 & 2,99 \\
3 & 0.8163 & 0.1836 & -52 & 100 & -34 & $-2,102$ \\
\hline
\end{tabular}

${ }^{\mathrm{a}} \mathrm{F}_{1}$ and ${ }^{\mathrm{b}} 1-\mathrm{F}_{1}$ are the fractions of PBuA and PMMA in the soft segment, respectively.

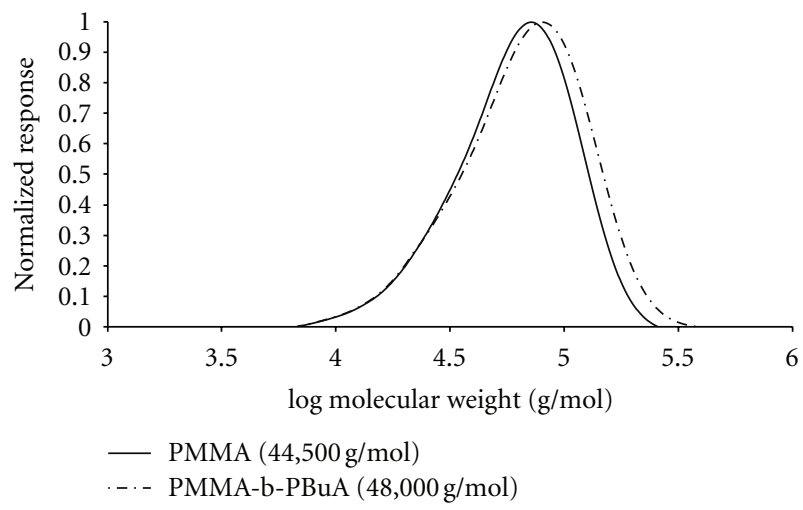

FIGURE 1: SEC analysis of a PMMA synthesized by RITP and a block copolymer synthesized by sequential copolymerization with BuA.

experimental values; in experiment 1 , for example, it was calculated to be $56000 \mathrm{~g} / \mathrm{mol}$ according to;

$$
\begin{aligned}
\mathrm{Mn}= & \frac{(\mathrm{g} \mathrm{BuA} * \text { conversion })+(\mathrm{g} \text { MMA } * \text { conversion })}{\text { moles of PMMA }} \\
& + \text { Mn of PMMA (experimental }) .
\end{aligned}
$$

Polydispersity indexes increased slightly after the block copolymerization which is typical in an RITP experiment due to the accumulation of dead chains that is unavoidable in any RDRP technique. Figure 1 shows the SEC curves of the macrochain transfer agent (PMMA) and the resulting diblock copolymer in experiment 1 . Both chromatograms show a monomodal distribution but the copolymer presents a shift toward higher molecular weights region, confirming that most of the growing PMMA chains remain in the living stage and take part in the formation of the diblock copolymer. A small fraction of PMMA with low molecular weight remains inactive during the copolymerization.

Composition of copolymers was determinate by ${ }^{1} \mathrm{H}$ NMR resulting in 93, 80, and $70 \mathrm{wt} \%$ of PMMA in experiments 1,2 , and 3, respectively; the rest for $100 \mathrm{wt} \%$, in each case, is constituted by PBuA. Most compositions give glassy properties to these materials, except the one with $70 \mathrm{wt} \%$ of PMMA. The presence of two shifted glass transition temperatures $\left(T_{g}\right)$ reinforces the statement of these being block copolymers. $T_{g}$ of pure $\mathrm{PBuA}$ is expected at $-52^{\circ} \mathrm{C}$; however the large shift to around $0^{\circ} \mathrm{C}$ observed in our materials can be explained considering the portion of PMMA in the second block (soft segment), in all cases (see Table 3 ). Comparing the experimental results with those calculated using the Fox equation [24] (4), a high deviation is observed as shown in Table 3, especially for the copolymers with 20 and $30 \mathrm{wt} \%$ of PBuA; however, according to Brostow et al. [25], this deviation is normal and it is explained because of the immiscibility of the components of soft segments; that is, the higher the miscibility, the closer the predicted $T_{g}$ to the experimental $T_{g}$ :

$$
\frac{1}{T_{g}}=\frac{F_{1}}{T_{g, 1}}+\frac{1-F_{1}}{T_{g, 2}}
$$

where $T_{g}$ is the soft segment $T_{g}$ prediction; $\mathrm{F}_{1}$ is the PBuA fraction in the soft segment; $T_{g, 1}$ is the $T_{g}$ of PBuA homopolymer; $T_{g, 2}$ is the $T_{g}$ of PMMA homopolymer.

To determine the effect of PBuA content on the mechanical properties of the diblock copolymers, DMA analysis was done. The storage modulus $\left(E^{\prime}\right)$ at $-20^{\circ} \mathrm{C}$ decreases from 4428,3846 , and $2739 \mathrm{MPa}$ for copolymers with 7, 20, and $30 \mathrm{wt} \%$ of $\mathrm{PBuA}$ content, respectively, as expected; this behavior is due to the rubbery nature of PBuA.

On the other hand, Figure 2(a) presents the variation of $\tan \delta\left(E^{\prime \prime} / E^{\prime}\right)$ with temperature for the three diblock copolymers. The ones with 30 and $20 \mathrm{wt} \%$ of PBuA show two transitions whereas the one with $7 \mathrm{wt} \%$ shows only one transition. Those transitions occur at $33 \pm 3$ and $100^{\circ} \mathrm{C}$ and correspond to the $\mathrm{P}(\mathrm{BuA}-c o-\mathrm{MMA})$ block (soft segment) and to the PMMA block (hard segment) in the copolymer, respectively. All transitions shift to high temperatures with respect to the $\mathrm{PBuA}$ homopolymer $\left(-52^{\circ} \mathrm{C}\right)$ and remain constant for the PMMA $\left(100^{\circ} \mathrm{C}\right)$; this behavior is explained due to the sequential formation of the second block $\mathrm{P}$ (BuAco-MMA) that represents the soft segment rich in PBuA but still containing PMMA. It was also observed that an increase in the PBuA content causes an increase in the area under the $\tan \delta$ curve that corresponds to the soft segment.

Figures 2(b) and 2(c) show the micrographs by TEM of the copolymers with 20 and $30 \mathrm{wt} \%$ of PBuA. In both cases, the presence of a PBuA-stained phase [22] (darker phase in the images) was observed, but the copolymer with $30 \mathrm{wt} \%$ of $\mathrm{PBuA}$ in Figure 2(c) presented a higher volume fraction of rubbery phase than the one with $20 \mathrm{wt} \%$ of $\mathrm{PBuA}$ in Figure 2(b). This coincides with the observed areas under the $\tan \delta$ curve, at ca. $T_{g}$ of the soft segments, which is greater for the sample with $30 \mathrm{wt} \%$ of $\mathrm{PBuA}$.

3.2. Nanostructured Films. Taking the synthesized copolymers, colloidal suspensions were prepared with $0.4-1.2 \mathrm{wt} \%$ of carboxyl functionalized MWCNT. These colloidal suspensions were deposited on glass cover slits and the drying rate $(N)$ was evaluated. Figure 3(a) shows the weight loss of films prepared from the solution of $\mathrm{CHCl}_{3} /$ bock copolymer with 


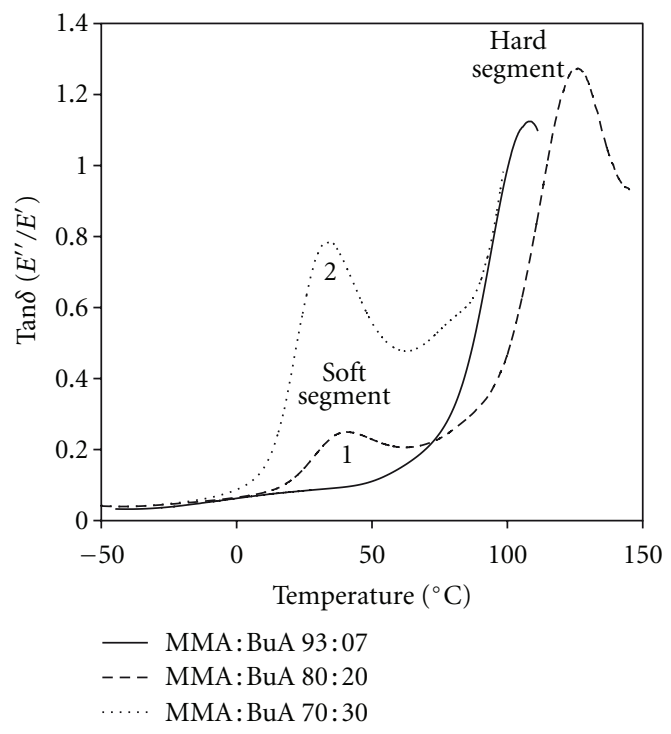

(a)
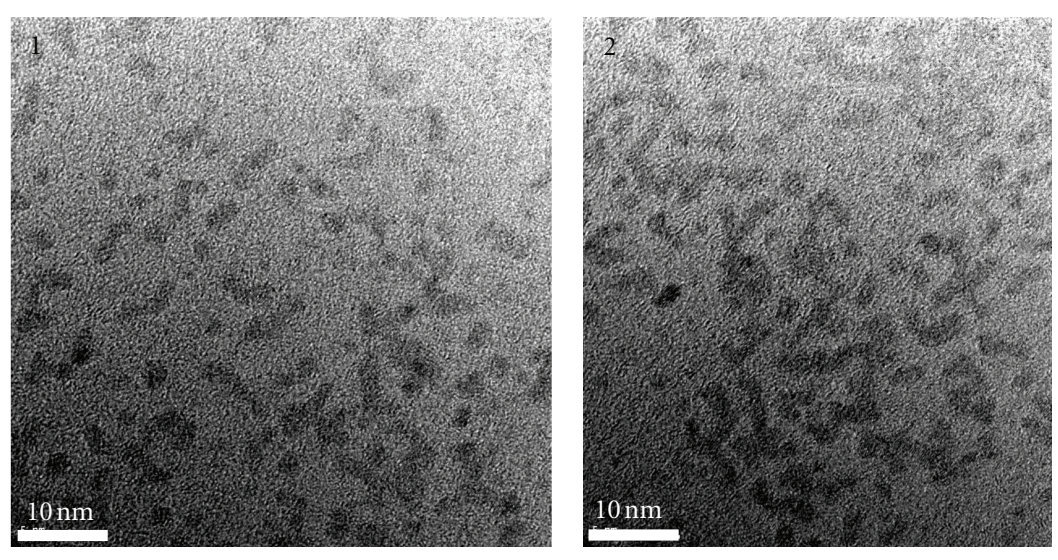

(b)

(c)

FIgure 2: (a) $\tan \delta\left(E^{\prime \prime} / E^{\prime}\right)$ for the synthesized copolymers, (b) TEM image for the copolymer with $20 \mathrm{wt} \%$ of PBuA, and (c) with $30 \mathrm{wt} \%$ of $\mathrm{PBuA}$ (scale bar at $10 \mathrm{~nm})$.

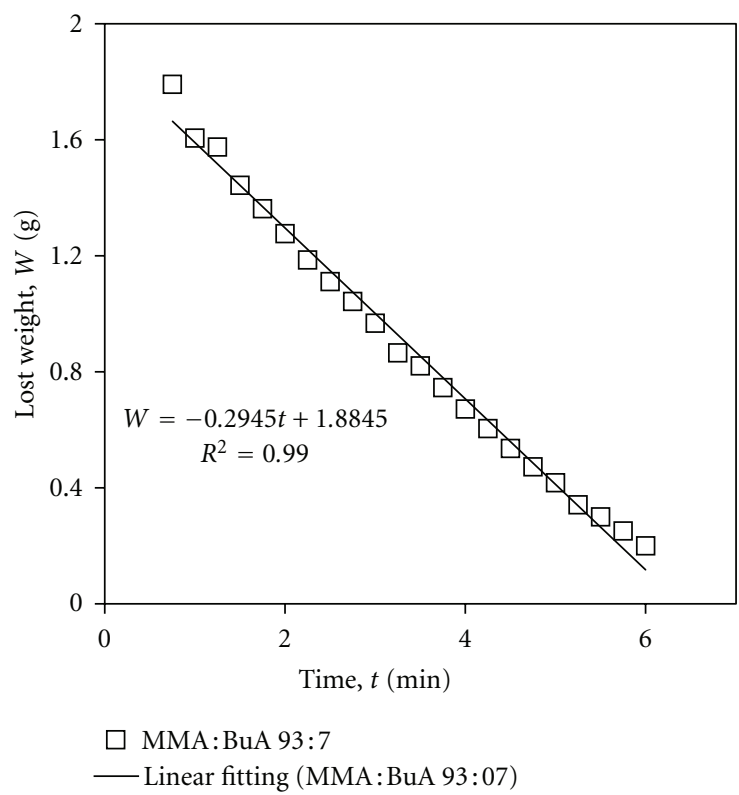

(a)

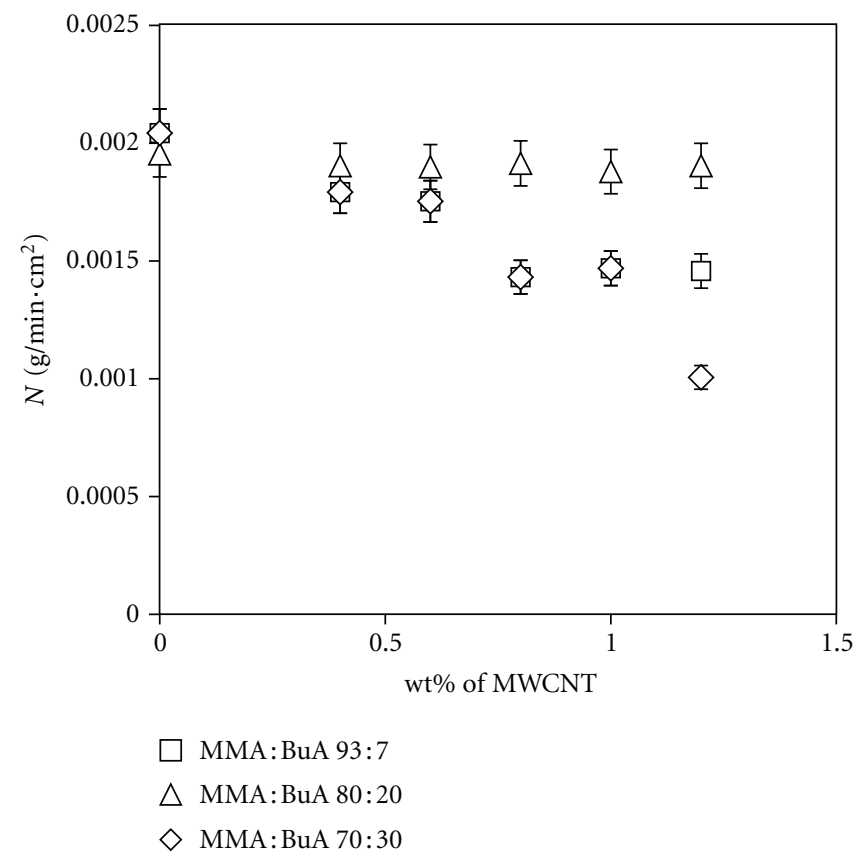

(b)

Figure 3: (a) The lost weight as a function of time for the solution $\mathrm{CHCl}_{3} /$ block copolymer with 7 wt $\%$ of $\mathrm{PBuA}$ and (b) $\mathrm{N}$ values for the suspensions $\mathrm{CHCl}_{3} /$ block copolymers (with 7, 20, and $30 \mathrm{wt} \%$ of PBuA)/MWCNT.

$7 \mathrm{wt} \%$ of $\mathrm{PBuA}$, where the $d w / d t$ was determined from the linear function of weight loss with time. In this case, $N$ was $0.203 \mathrm{~g} / \mathrm{min} \cdot \mathrm{cm}^{2}$. Similarly, the $N$ values were calculated for the other colloidal suspensions. Figure 3(b) shows the $N$ values for the $\mathrm{CHCl}_{3} /$ block copolymer/MWCNT solutions with 7,20 , and $30 \mathrm{wt} \%$ of $\mathrm{PBuA}$ with $0.4-1.2 \mathrm{wt} \%$ of functionalized MWCNT, as a function of MWCNT concentration.
When comparing the pure copolymers, the solvent evaporation or film drying rate $(N)$ of all three copolymer compositions studied is similar at low CNT contents; however, as the CNT content increases, $N$ decreases, especially for those copolymers with $30 \mathrm{wt} \%$ of $\mathrm{PBuA}$.

In all three cases however, the drying rate $(N)$ decreases with increasing MWCNT content. This decrease is more pronounced for those compositions with 7 and $30 \mathrm{wt} \%$ of PBuA. 


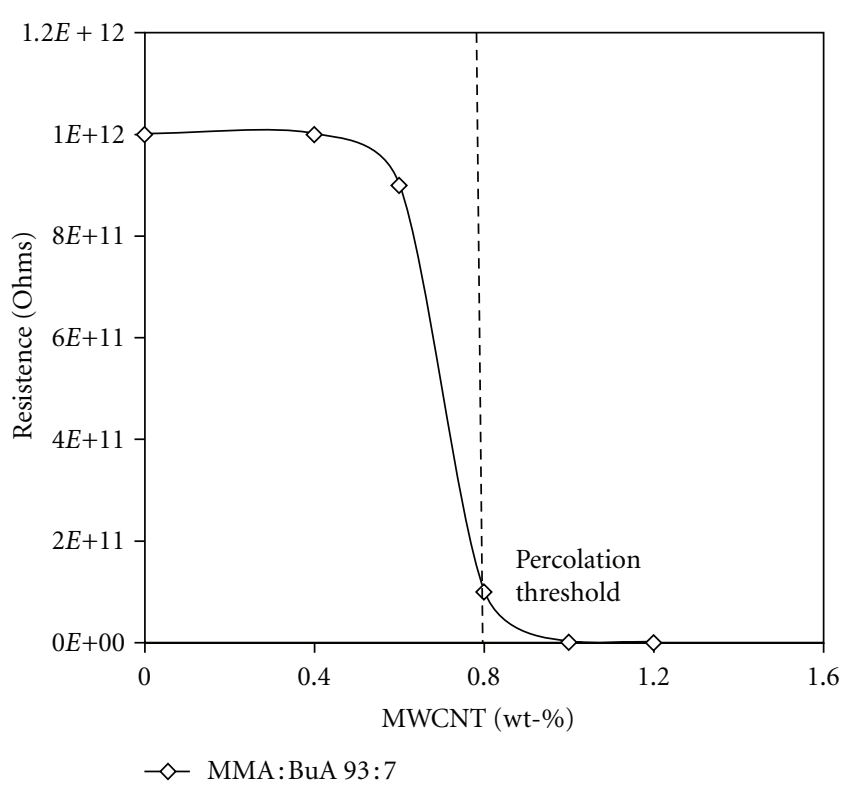

Figure 4: Electrical resistance of the nanostructured films as a function of the MWCNT content.

3.3. Nanocomposites Electric Resistance. Figure 4 shows the variation of the electrical resistance with respect to the MWCNT content, for the copolymer with $7 \mathrm{wt} \%$ of $\mathrm{PBuA}$. A drastic decrease in the resistance from $1 \times 10^{12}$ to $1 \times$ $10^{6} \mathrm{Ohms}$ is observed at $0.8 \mathrm{wt} \%$ of MWCNT, which would indicate the concentration at the percolation threshold for this compound. Similar behavior was observed for the copolymers with 20 and $30 \mathrm{wt} \%$ of PBuA that reached a resistance of $1 \times 10^{6} \mathrm{Ohms}$ at the same content of MWCNT.

\subsection{Dynamic-Mechanic Properties of Nanostructures Films.} Figure 5 shows the DMA analysis for the block copolymers with $0.8 \mathrm{wt} \%$ of MWCNT, which is the concentration at the percolation threshold. As expected the storage modulus of the copolymers with MWCNT, at $-20^{\circ} \mathrm{C}$, increases from 3121 to 4331 and finally to $5358 \mathrm{MPa}$, for the copolymers at 30, 20, and $7 \mathrm{wt} \%$ of $\mathrm{PBuA}$, respectively. This result can be attributed to the decreasing content of the elastomeric PBuA content in the copolymer at constant MWCNT content. Comparing these $E^{\prime}$ values with those corresponding to the pure copolymers, the storage modulus increases from 11 to $17 \%$ as the PBuA content decreases.

Considering, the intended application of the nanocomposites to be as a coating for textiles, the variation in modulus can be regulated by the PBuA content in the synthesized poly(methyl methacrylate)- $b$-poly(butyl acrylate-co-methyl methacrylate) copolymers. For example, the incorporation of $0.8 \mathrm{wt} \%$ of MWCNT in a block copolymer with a concentration of $30 \mathrm{wt} \%$ of $\mathrm{PBuA}$ can result in a lower storage modulus $(3639 \mathrm{MPa})$, in respect of a block copolymer with $20 \mathrm{wt} \%$ of PBuA (4041 MPa). On the other hand, apparently there is a decrease in $T_{g}$ (from Figure $2(\mathrm{a})$ ) from $30-35^{\circ} \mathrm{C}$, for the pure copolymers, to $21-20^{\circ} \mathrm{C}$ for the nanocomposites at 7 and $20 \mathrm{wt} \%$ of $\mathrm{PBuA}$, respectively. This behavior is attributed to the nanocomposites preparation method (casting), which

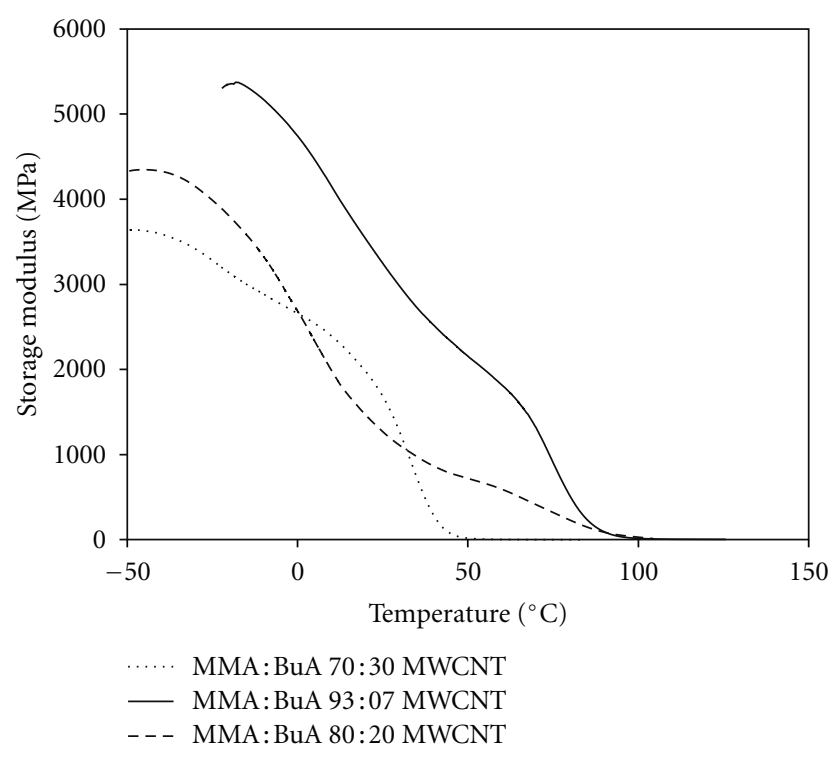

FIGURE 5: DMA analysis for the block copolymers/MWCNT nanocomposites with $8 \mathrm{wt} \%$ of MWCNT.

allows a slow arrangement of the polymer chains so that the PBuA rich block, in the copolymer, will present more interaction with itself, resulting in a larger dispersed phase. Apparently, it tends to behave as a polymer blend; however, those transitions are still far from the values for the PBuA (ca. $\left.-500^{\circ} \mathrm{C}\right)$.

Figure 6 presents the SEM micrographs of the nanocomposites prepared with the three different copolymers at $0.8 \mathrm{wt} \%$ of MWCNT. In all three cases, a good homogeneous distribution and dispersion is apparent. However, after a thorough analysis, a clear tendency to agglomerate can be observed (dots 1, 2, and 3 in Figures 6(b) and 6(c)). This tendency seems to increase with increasing PBuA content in the copolymers. This can be attributed to poor compatibility between the copolymers and the CNT, which becomes poorer with increasing the PBuA content.

Also, the presence of two distinctive phases can be observed in copolymers with 7 and $30 \mathrm{wt} \%$ of PBuA in Figures 6(a) and 6(c). In the first case, we have a dispersed discrete phase as droplets with an average diameter of $0.5 \mathrm{~mm}$, whereas in the second case, no distinction can be observed between the two components, which could indicate that at $30 \mathrm{wt} \%$ of $\mathrm{PBuA}$, there is a type of cocontinuity of phases.

In this sense, it appears that the MWCNTs have a preference to locate at the PMMA pure block and/or in the interface, which causes a well distribution but poor dispersion in the copolymers with higher PBuA content. It results in the formation of tridimensional network of MWCNT (by bridging effect) [26] which favors the drastic decrease in the surface resistance at $8 \mathrm{wt} \%$ of MWCNT.

\section{Conclusions}

Randomized block copolymers of poly(methyl methacrylate)- $b$-poly(butyl acrylate-co-methyl methacrylate) were 


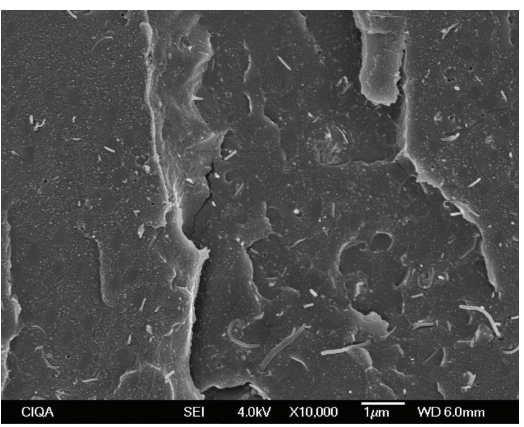

(a)

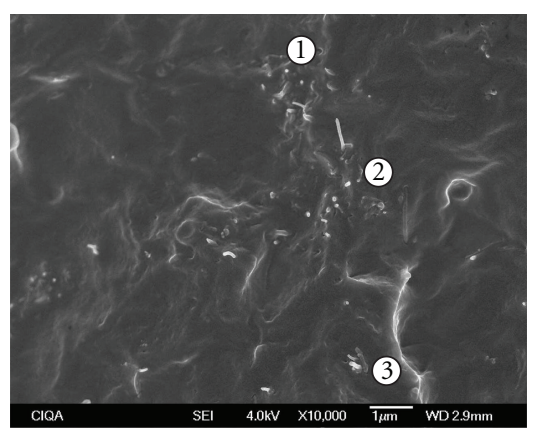

(b)

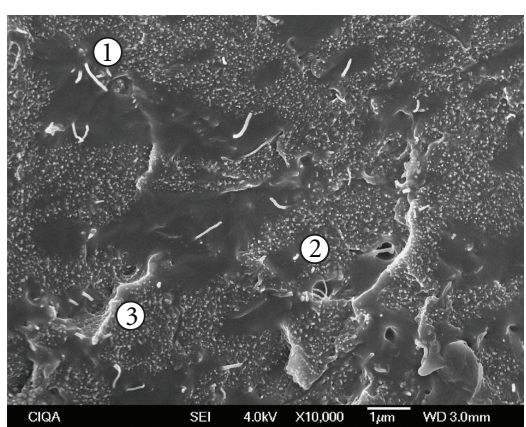

(c)

FIGURE 6: SEM images of block copolymers/MWCNT nanocomposites with 8 wt $\%$ of nanotubes with (a) 7, (b) 20, and (c) 30 wt $\%$ of PBuA.

successfully synthesized by the RITP technique, which showed, in terms of dynamic-mechanic properties, low storage modulus with the $\mathrm{PBuA}$ content increase. It was observed that an increase in the PBuA content results in a decrease in the drying rate $(N)$, of the different colloidal suspensions. In terms of electrical resistance for the nanocomposites, the $\mathrm{PBuA}$ content did not show a significant effect. On the other hand, the dispersion and distribution of MWCNT into the copolymer matrix were confirmed by SEM. An increase in the PBuA content causes more interaction between the nanotubes themselves, presenting a good distribution but a poor dispersion which induces the percolation threshold.

\section{Acknowledgments}

The authors would like to thank Francisco Zendejo, Jose López Rivera, and Guadalupe Mendez for their technical support in the preparation and characterization of colloidal suspensions and nanostructured films and Judith Cabello for her help in ${ }^{1} \mathrm{H}$ NMR analysis. The authors also thank CONACYT for its financial support to carry out this study through projects 84424 and 146970.

\section{References}

[1] S. Iijima, "Helical microtubules of graphitic carbon," Nature, vol. 354, no. 6348, pp. 56-58, 1991.

[2] S. Abbasi, A. Derdouri, and P. J. Carreau, "Properties of microinjection molding of polymer multiwalled carbon nanotube conducting composites," Polymer Engineering and Science, vol. 51, no. 5, pp. 992-1003, 2011.

[3] S. Yesil and G. Bayram, "Poly(ethylene terephthalate)/carbon nanotube composites prepared with chemically treated carbon nanotubes," Polymer Engineering and Science, vol. 51, no. 7, pp. 1286-1300, 2011.

[4] Y. Zhao, Z. Qiu, S. Yan, and W. Yang, "Crystallization behavior of biodegradable poly(L-lactide)/multiwalled carbon nanotubes nanocomposites from the amorphous state," Polymer Engineering and Science, vol. 51, no. 8, pp. 1564-1573, 2011.

[5] V. J. Cruz-Delgado, M. E. Esparza-Juárez, B. L. EspañaSánchez,, M. T. Rodriguez Hernández, and C. A. ÁvilaOrta, "Nanocompuestos poliméricos semiconductores de PET/MWCNT: preparación y caracterización," Superficies y Vacio, vol. 20, no. 2, pp. 6-11, 2007.
[6] A. Khosla and B. L. Gray, "Preparation, micro-patterning and electrical characterization of functionalized carbon-nanotube polydimethylsiloxane nanocomposite polymer," Macromolecular Symposia, vol. 297, no. 1, pp. 210-218, 2010.

[7] B. Lee and G. Dai, "Influence of interfacial modification on the thermal conductivity of polymer composites," Journal of Materials Science, vol. 44, no. 18, pp. 4848-4855, 2009.

[8] K. A. Davis and K. Matyjaszewski, Statistical, Gradient, Block and Graft Copolymers by Controlled/Living Radical Polymerizations, Springer, Berlin, Germany, 1st edition, 2002.

[9] D. Roy, J. T. Guthrie, and S. Perrier, "Graft polymerization: grafting poly(styrene) from cellulose via Reversible Addition-Fragmentation Chain Transfer (RAFT) polymerization," Macromolecules, vol. 38, no. 25, pp. 10363-10372, 2005.

[10] T. N. T. Phan, S. Maiez-Tribut, J. P. Pascault et al., "Synthesis and characterizations of block copolymer of poly(n-butyl acrylate) and gradient poly(methyl methacrylate-co-N,Ndimethyl acrylamide) made via nitroxide-mediated controlled radical polymerization," Macromolecules, vol. 40, no. 13, pp. 4516-4523, 2007.

[11] C. J. Hawker, A. W. Bosman, and E. Harth, "New polymer synthesis by nitroxide mediated living radical polymerizations," Chemical Reviews, vol. 101, no. 12, pp. 3661-3688, 2001.

[12] N. V. Tsarevsky and K. Matyjaszewski, “”Green:atom transfer radical polymerization: from process design to preparation of well-defined environmentally friendly polymeric materials," Chemical Reviews, vol. 107, no. 6, pp. 2270-2299, 2007.

[13] G. Moad, E. Rizzardo, and S. H. Thang, "Living radical polymerization by the RAFT process-a first update," Australian Journal of Chemistry, vol. 59, no. 10, pp. 669-692, 2006.

[14] P. Lacroix-Desmazes, R. Severac, and B. Boutevin, "Reverse iodine transfer polymerization of methyl acrylate and n-butyl acrylate," Macromolecules, vol. 38, no. 15, pp. 6299-6309, 2005.

[15] P. Lacroix-Desmazes, J. Tonnar, and B. Boutevin, "Reverse iodine transfer polymerization (RITP) in emulsion," Macromolecular Symposia, vol. 248, pp. 150-157, 2007.

[16] F. J. Enríquez-Medrano, R. Guerrero-Santos, M. HernándezValdez, and P. Lacroix-Desmazes, "Synthesis of diblock and triblock copolymers from butyl acrylate and styrene by reverse iodine transfer polymerization," Journal of Applied Polymer Science, vol. 119, no. 4, pp. 2476-2484, 2011.

[17] D. Rayeroux, B. N. Patra, and P. Lacroix-Desmazes, "Synthesis of amphiphilic diblock copolymers of polystyrene and poly(Acrylic Acid) by reverse iodine transfer polymerization (RITP) in solution and emulsion," Polymer Preprints, vol. 52, no. 2 , p. $715,2011$. 
[18] B. N. Patra, D. Rayeroux, and P. Lacroix-Desmazes, "Synthesis of cationic amphiphilic diblock copolymers of poly(vinylbenzyl triethylammonium chloride) and polystyrene by reverse iodine transfer polymerization (RITP)," Reactive and Functional Polymers, vol. 70, no. 7, pp. 408-413, 2010.

[19] C. Boyer, P. Lacroix-Desmazes, J. J. Robin, and B. Boutevin, "Reverse iodine transfer polymerization (RITP) of methyl methacrylate," Macromolecules, vol. 39, no. 12, pp. 4044-4053, 2006.

[20] P. Lacroix-Desmazes, D. Rayeroux, and M. Villa-Hernandez, "Reverse iodine transfer polymerization (RITP): from kinetics and mechanisms to macromolecular engineering," Polymer Preprints, vol. 52, no. 2, p. 582, 2011.

[21] M. A. Villa-Hernandez, F. J. Enriquez-Medrano, R. GuerreroSantos, and P. Lacroix-Desmazes, "Use of difunctional initiator in reverse iodine transfer polymerization (RITP)," Polymer Preprints, vol. 52, no. 2, p. 590, 2011.

[22] L. C. Sawyer and D. T. Grubb, Polymer Microscopy, Champman and Hall, 1st edition, 1987.

[23] W. McCabe, J. Smith, and P. Harriot, Unit Operations of Chemical Engineering, McGraw Hill, 7th edition, 2005.

[24] T. G. Fox, "Influence of diluent and copolymer compositionon the glass transition temperature of a polymer system," Bulletin of the American Physical Society, vol. 1, p. 123, 1956.

[25] W. Brostow, R. Chiu, I. M. Kalogeras, and A. Vassilikou-Dova, "Prediction of glass transition temperatures: binary blends and copolymers," Materials Letters, vol. 62, no. 17-18, pp. 3152-3155, 2008.

[26] J. M. Margolis, Conductive Polymers and Plastics, vol. 1st, Champman and Hall, 1989. 

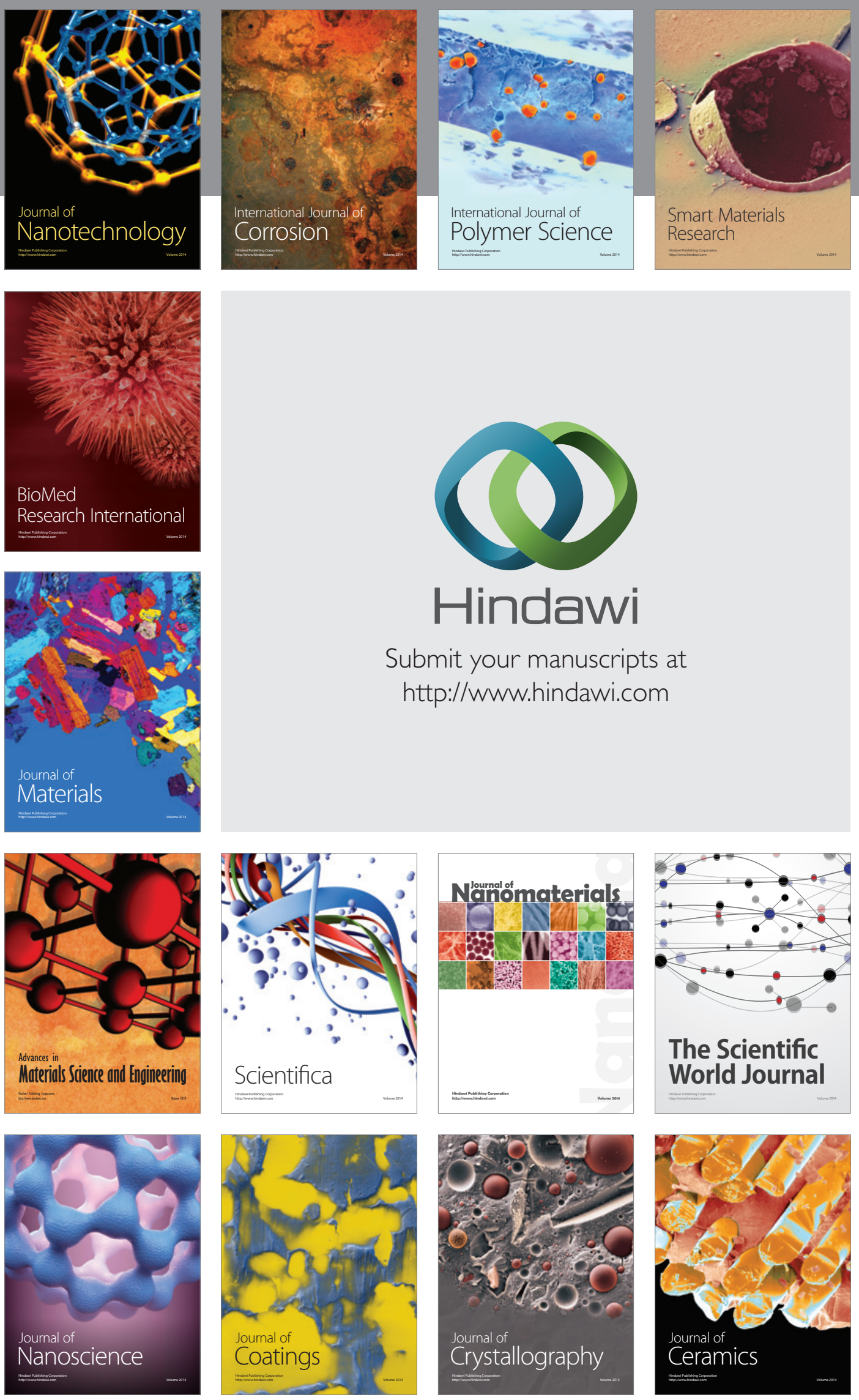

The Scientific World Journal

Submit your manuscripts at

http://www.hindawi.com

\section{World Journal}

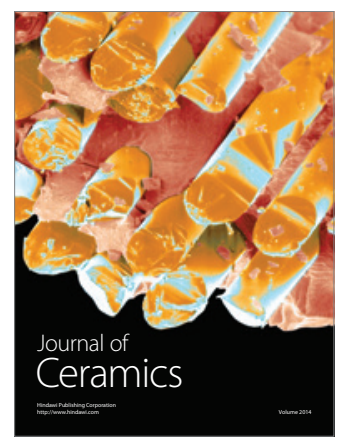

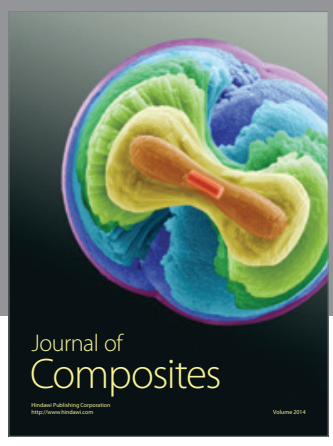
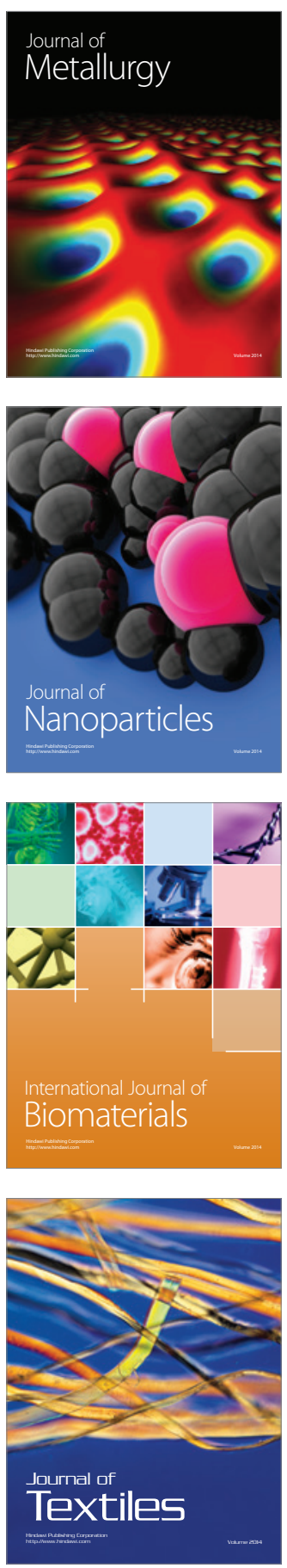